\title{
Rational Approximations to the Incomplete Elliptic Integrals of the First and Second Kinds*
}

\section{By Wyman G. Fair and Yudell L. Luke}

In this note we derive rational approximations (in Eqs. (20) and (21) below) to the integrals

$$
F(\varphi, k)=\int_{0}^{\varphi}\left(1-k^{2} \sin ^{2} t\right)^{-1 / 2} d t
$$

and

$$
E(\varphi, k)=\int_{0}^{\varphi}\left(1-k^{2} \sin ^{2} t\right)^{1 / 2} d t
$$

where $k^{2}$ is real and $0<\varphi<\pi / 2$, by obtaining the main diagonal Padé approximations to closely related functions. It is sufficient to consider the case $0<k^{2}<1$, for if $k^{2}>1$,

$$
\begin{gathered}
F(\varphi, k)=k_{1} F\left(\beta_{1}, k_{1}\right) \text { and } E(\varphi, k)=k_{1}\left[E\left(\beta_{1}, k_{1}\right)+\left(1-k^{2}\right)^{2} F\left(\beta_{1}, k_{1}\right)\right], \\
k_{1}=1 / k \text { and } \beta_{1}=\arcsin (k \sin \varphi),
\end{gathered}
$$

while if $k^{2}<0$,

$$
\begin{aligned}
F(\varphi, k) & =\left(1-{k_{2}}^{2}\right)^{1 / 2} F\left(\beta_{2}, k_{2}\right) \quad \text { and } \\
E(\varphi, k) & =\left(1-{k_{2}}^{2}\right)^{-1 / 2}\left[E\left(\beta_{2}, k_{2}\right)-\frac{k_{2}{ }^{2} \sin \beta_{2} \cos \beta_{2}}{\left(1-{k_{2}}^{2} \sin ^{2} \beta_{2}\right)^{1 / 2}}\right], \\
k_{2} & =|k|\left(1-k^{2}\right)^{-1 / 2} \text { and } \beta_{2}=\arcsin \left[\left(\frac{1-k^{2}}{1-k^{2} \sin ^{2} \varphi}\right)^{1 / 2} \sin \varphi\right] .
\end{aligned}
$$

Define $m=k^{2}$ and

$$
\begin{array}{rlrl}
a & =\left[\frac{(2-m)^{2}}{1+m}\right]^{1 / 3}>0, & b & =\left[\frac{(1-2 m)^{3}}{(m-2)(m+1)}\right]^{1 / 3}, \\
c & =\left[\frac{(1+m)^{2}}{m-2}\right]^{1 / 3}<0, & x & =c+\frac{a-c}{\sin ^{2} \varphi}, \\
h & =a\left[c+\frac{b(2 m-1)}{m-2}\right]<0, & g & =2 m-1, \quad s=2\left[\frac{2-m}{3 a}\right]^{1 / 2}, \\
r(x) & =x^{3}+h x+g, & v(x) & =\frac{(x-c)^{3}(x-a)}{x-b}, \\
I_{1}(x) & =\int_{x}^{\infty}[r(t)]^{-1 / 2} d t \quad \text { and } \quad I_{2}(x)=\int_{x}^{\infty}[v(t)]^{-1 / 2} d t .
\end{array}
$$

Received February 3, 1967.

* This work was supported by the National Aeronautics and Space Administration under Contract No. NASA Hq. 80X0108(64). 
Then $a>b>c$ are the real roots of $r(z)=0$ and it follows from [1] that

$$
F(\varphi, k)=s^{-1} I_{1}(x) \text { and } E(\varphi, k)=s^{-1} I_{2}(x) .
$$

Set

$$
G_{1}(x)=[r(x)]^{1 / 2} I_{1}(x), \quad G_{2}(x)=\frac{[v(x)]^{1 / 2}}{2 x} I_{2}(x) .
$$

Then $G_{l}(x)(l=1,2)$ satisfies the differential equation

$$
r(x) \gamma_{l}(x) G_{l}^{\prime}(x)-\delta_{l}(x) G_{l}(x)+r(x)=0,
$$

where

$$
\begin{aligned}
& \gamma_{1}(x)=1, \quad \gamma_{2}(x)=2 x, \quad \delta_{1}(x)=\frac{1}{2}\left(3 x^{2}+h\right), \\
& \delta_{2}(x)=x^{3}-2(a+2 b) x^{2}+(a b-b c-3 a c) x+2 a b c .
\end{aligned}
$$

For convenience, we make the transformations

$$
z=1 / x, \quad G_{1}(z)=x^{-1}\left[2+x^{2} H_{1}(x)\right], \quad G_{2}(z)=H_{2}(z) .
$$

Then (8) becomes

$$
\eta_{l}(x) H_{l}^{\prime}(x)+\rho_{l}(x) H_{l}(x)+\xi_{l}(x)=0, \quad l=1,2,
$$

where

$$
\begin{aligned}
& \eta_{1}(x)=x\left(1+h x^{2}+g x^{3}\right), \quad \eta_{2}(x)=2 \eta_{1}(x), \quad \rho_{1}(x)=\frac{5}{2}+\frac{3 h}{2} x^{2}+g x^{3}, \\
& \rho_{2}(x)=1-2(a+2 b) x+(a b-b c-3 a c) x^{2}-2 g x^{3}, \quad \xi_{1}(x)=-2 h-3 g x, \\
& \xi_{2}(x)=-1-h x^{2}-g x^{3}, \quad H_{1}(0)=\frac{4 h}{5} \text { and } \quad H_{2}(0)=1 .
\end{aligned}
$$

Main diagonal Padé approximations for the solution to (10) are readily computed by using the results of [2]. For completeness we list the recurrence relations which determine the main diagonal Padé approximations to $H_{l}(x), l=1,2$. In the notation of [2], we have: for $l=1$;

$$
\begin{aligned}
y(x) & =H_{1}(x), \\
y_{0} & =y(0)=4 h / 5, \\
p_{0} & =p_{2}=0, \quad p_{1}=1, \quad p_{3}=h, \quad p_{4}=g, \\
q_{0} & =5 / 2, \quad q_{1}=0, \quad q_{2}=3 h / 2, \quad q_{3}=g, \\
s_{0} & =-2 h, \quad s_{1}=-3 g, \quad s_{2}=s_{3}=0,
\end{aligned}
$$

and for $l=2, y(x)=H_{2}(x)$,

$$
\begin{aligned}
y_{0} & =y(0)=1, \\
p_{0} & =p_{2}=0, \quad p_{1}=2, \quad p_{3}=2 h, \quad p_{4}=2 g, \\
\text { (12) } q_{0} & =1, \quad q_{1}=-2(a+2 b), \quad q_{2}=a b-b c-3 a c, \quad q_{3}=-2 g, \\
s_{0} & =-1, \quad s_{1}=0, \quad s_{2}=-h, \quad s_{3}=-g .
\end{aligned}
$$

Let 


$$
y_{n}=\frac{A_{n}}{B_{n}}, \quad A_{n}=\sum_{k=0}^{n} a_{n, k} x^{k}, \quad B_{n}=\sum_{k=0}^{n} b_{n, k} x^{k}
$$

be the $n$ th-order main diagonal Padé approximations to $y(x)$. Then $A_{n}$ and $B_{n}$ satisfy

$$
A_{n}=\left(1+\beta_{n} x\right) A_{n-1}+\alpha_{n} x^{2} A_{n-2} .
$$

The equations which determine $\alpha_{n}$ and $\beta_{n}$ are

$$
\alpha_{n}=-\tau_{n-1,1}\left[(-1)^{n} \alpha_{n-1,1}^{n} p_{1}+\alpha_{n-1,2} u_{1}+2 \sum_{j=3}^{n} \alpha_{n-1, j} \tau_{j-2,1}\right]^{-1},
$$

and

$$
\begin{aligned}
\beta_{n}= & -\left[\tau_{n-1,2}+\alpha_{n, 2} u_{2}+2 \sum_{j=3}^{n} \alpha_{n, j}\left(\tau_{j-2,2}+\beta_{j-1} \tau_{j-2,1}\right)\right] \\
& \times\left[2 \tau_{n-1,1}+\alpha_{n, 2} u_{1}+2 \sum_{j=3}^{n} \alpha_{n, j} \tau_{j-2,1}\right]^{-1}, \quad n=2,3,4 \cdots,
\end{aligned}
$$

where

$$
\begin{aligned}
& \tau_{n, k}=\tau_{n-1, k+2}+2 \beta_{n} \tau_{n-1, k+1}+\alpha_{n}{ }^{2} \tau_{n-2, k}+\beta_{n}{ }^{2} \tau_{n-1, k}+(-1)^{n} \alpha_{n, 1} p_{k+2} \\
& +\alpha_{n, 2} u_{k+2}+\alpha_{n, 2} \beta_{n} u_{k+1} \\
& +2 \sum_{j=3}^{n} \alpha_{n, j}\left[\tau_{j-2, k+2}+\left(\beta_{n}+\beta_{j-1}\right) \tau_{j-2, k+1}+\beta_{n} \beta_{j-1} \tau_{j-2, k}\right], \\
& n=2,3,4, \cdots, k=1,2,3, \\
& u_{k}=2 y_{0} q_{k}+2 s_{k}+\left(a_{1,1}+b_{1,1} y_{0}\right) q_{k-1}+2 b_{1,1} s_{k-1} \quad k=1,2,3,4, \\
& \alpha_{k, j}=\alpha_{k} \alpha_{k-1} \cdots \alpha_{j}, \alpha_{k, k}=\alpha_{k}, \alpha_{k-1, k}=1 \text { and } \alpha_{k, j}=0, \quad k<j-1 .
\end{aligned}
$$

The starting values for computation are

$$
\begin{aligned}
\tau_{0, k}= & y_{0} q_{k}+s_{k}, \\
\tau_{1 k}= & -\alpha_{1} p_{k+2}+y_{0} q_{k+2}+s_{k+2}+\left(a_{1,1}+b_{1,1} y_{0}\right) q_{k+1} \\
& +2 b_{1,1} s_{k+1}+a_{1,1} b_{1,1} q_{k}+b_{1,1}^{2} s_{k}, \quad k=1,2,3
\end{aligned}
$$

for $l=1$,

$$
\begin{aligned}
\alpha_{1} & =-6 g / 7, \quad \beta_{1}=b_{1,1} \\
a_{1,1} & =6 g / 7+\frac{56 h^{3}}{225 g}, \quad b_{1,1}=\frac{14 h^{2}}{45 g}
\end{aligned}
$$

for $l=2$,

$$
\begin{aligned}
\alpha_{1} & =-2 / 3(a+2 b), \quad \beta_{1}=b_{1,1}, \\
a_{1,1} & =\frac{4 a^{2}+16 b^{2}+25 a b-9 b c-27 a c-9 h}{30(a+2 b)}, \\
b_{1,1} & =-\frac{4 a^{2}-16 b^{2}-19 a b+3 b c+9 a c-3 h}{10(a+2 b)} .
\end{aligned}
$$

In either case, we have 


$$
A_{0}=y_{0}, \quad A_{1}=y_{0}+a_{1,1} x, \quad B_{0}=1, \quad B_{1}=1+b_{1,1} x .
$$

Thus, rational approximations to the incomplete elliptic integrals of the first and second kind respectively are

$$
F_{n}(\varphi, k)=\frac{[r(x)]^{1 / 2}}{s}\left[2 x+\frac{A_{n}(1 / x)}{x B_{n}(1 / x)}\right]
$$

and

$$
E_{n}(\varphi, k)=\frac{2 x[v(x)]^{-1 / 2}}{s} \frac{A_{n}(1 / x)}{B_{n}(1 / x)} .
$$

In the special case, $k^{2}=m=\frac{1}{2}$, the approximation (20) does not apply. However, since $g=0$ in this case, (20) becomes

(22) $t(1+h t) H_{1}{ }^{\prime}(t)+\frac{1}{4}(5+3 h t) H_{1}(t)-h=0, \quad H_{1}(0)=4 h / 5, \quad t=x^{2}$, and $H_{1}(t)=(4 h / 5){ }_{2} F_{1}(1,3 / 4 ; 9 / 4 ;-h t)$ is the solution to $(22)$. Padé approximations to this hypergeometric function together with an error analysis are available in [3].

Numerical results indicate rapid convergence of the approximations (20) and (21). These approximations are evidently insensitive to changes in $k^{2}$ and are very powerful for $\varphi<\pi / 3$. They weaken as $\varphi$ approaches $\pi / 2$; however, the Landen transformations

where

$$
\begin{aligned}
& F(\varphi, k)=\frac{2}{1+k} F\left(\varphi_{1}, k_{1}\right), \\
& E(\varphi, k)=(1+k) E\left(\varphi_{1}, k_{1}\right)+(1-k) F\left(\varphi_{1}, k_{1}\right)-k \sin \varphi
\end{aligned}
$$

$$
k_{1}=2 \sqrt{ } k /(1+k) \text { and } \varphi_{1}=\frac{1}{2} \varphi+\frac{1}{2} \arcsin (k \sin \varphi),
$$

should reduce $\varphi$ to the desirable range in all but the extreme cases. For example, if $k=\frac{1}{2}$ and $\varphi=\pi / 2$ we have

$$
F\left(\frac{1}{2}, \pi / 2\right)=\frac{4}{3} F(2 \sqrt{ } 2 / 3, \pi / 3) .
$$

The approximations $\frac{4}{3} F_{n}(2 \sqrt{ } 2 / 3, \pi / 3)$ are listed in Table I.

TABLE I

\begin{tabular}{r|cc}
\hline$n$ & \multicolumn{2}{|c}{$\frac{4}{3} F_{n}$} \\
\hline 4 & 1.68579 & 32446 \\
6 & 1.68575 & 05579 \\
8 & 1.68575 & 03557 \\
10 & 1.68575 & 03548 \\
12 & 1.68575 & 03548 \\
\hline
\end{tabular}

The true value is 1.6857503548 .

We present in Table II a tabulation of $\epsilon_{n}=\left|F(\varphi, k)-F_{n}(\varphi, k)\right|$ for a number of values of $n, \varphi$ and $k$. The behavior of the error involved in approximating $E(\varphi, k)$ 
TABLE II

$k^{2}=.25$

\begin{tabular}{c|c|c|c|c|c}
\hline$\varphi \backslash n$ & 4 & 6 & 8 & 10 & 20 \\
\hline $60^{\circ}$ & $1.89(-5)^{*}$ & $4.1(-7)$ & $4.0(-8)$ & & \\
$80^{\circ}$ & $7.33(-2)$ & $2.11(-2)$ & $7.11(-3)$ & $3.84(-3)$ & $8.1(-7)$ \\
\hline
\end{tabular}

by $E_{n}(\varphi, k)$ is almost identical and so is omitted. In both tables $\epsilon_{n}<1.0 \times 10^{-8}$ for $\varphi \leq 30^{\circ}$ and $n \geq 4$ ( $k$ arbitrary) so that these values are not listed. No entry in the table signifies an error less than $1.0 \times 10^{-8}$.

$$
k^{2}=.75
$$

\begin{tabular}{c|c|c|c|c|c}
\hline$\varphi \backslash n$ & 4 & 6 & 8 & 10 & 20 \\
\hline $60^{\circ}$ & $1.92(-3)$ & $4.7(-7)$ & & & \\
$80^{\circ}$ & $1.51(-1)$ & $2.55(-2)$ & $5.44(-3)$ & $1.13(-3)$ & $8.0(-7)$ \\
\hline
\end{tabular}

* The number in parentheses indicates the power of ten by which the tabular entry is to be multiplied.

The authors thank John Nelson who wrote the FORTRAN program for the computations. This program is available from the authors on request.

Midwest Research Institute

Kansas City, Missouri 64110

1. P. F. Byrd \& M. D. Friedman, Handbook of Elliptic Integrals for Engineers and Physicists, Die Grundlehren der mathematischen Wissenschaften, Band 67, Springer-Verlag, Berlin, 1954. MR 15, 702.

2. W. FAIR, "Padé approximation to the solution of the Riccati equation," Math. Comp., v. 18,1964, pp. $627-634$. MR $29 \# 6630$.

3. Y. L. Luke, "The Padé table and the $\tau$-method," J. Math. Phys., v. 37, 1958, pp. 110127. MR $20 \# 5558$. 